\title{
Performance comparison between LTE-V2X and ITS-G5 under realistic urban scenarios
}

\author{
Mouna Karoui, Antonio Freitas, Gerard Chalhoub \\ LIMOS-CNRS - University Clermont Auvergne \\ \{mouna.karoui, antonio.freitas, gerard.chalhoub\}@uca.fr
}

\begin{abstract}
Wireless communications are being used for enhancing transport systems .In order to achieve these goals, some communication technologies are specially designed to support Vehicleto-everything (V2X) functionalities. C-V2X (Cellular Vehicle-toeverything) or LTE-V2X and ITS-G5 are one of the existing solutions respectively standardized by 3GPP (3rd Generation Partnership Project) and ETSI (European Telecommunications Standards Institute). While ITS-G5 is a dedicated wireless network for C-ITS applications, LTE-V2X shares the network resources with other LTE applications used on mobile devices. In this paper, we give an insight on both technologies by briefly describing their communication mechanisms. Then, we compare their performances under different use cases using a networking and vehicular simulation platform. Results show that ITS-G5 outperforms LTE-V2X (mode 3 ) in case of the presence of concurrent LTE data traffic with V2X service. Throughout the conducted scenarios, we demonstrate the negative impact of handover on LTE-V2X eNB scheduled mode.
\end{abstract}

Keywords-C-ITS, LTE-V2X, IEEE 802.11p/ITS-G5, End-toend delay.

\section{INTRODUCTION}

Emerging communication technologies present one of today's main interest in Cooperative Intelligent Transportation Systems (C-ITS) research field. Indeed, this ecosystem of communication technologies is necessary in order to offer better support to aspects such as safety, user comfort, traffic efficiency and environment friendliness.

Different solutions were proposed in the last few years mainly based on two categories : (i) Short range/ad-hoc-based and (ii) long range/cellular-based. These two key technologies are able to ensure various services between different road users.

These communication technologies also present an important support for vehicular networking performance enabling different architectural components to efficiently communicate with one another. Infrastructure-to-vehicle (I2V) Vehicle-toVehicle (V2V), and Vehicle-to-infrastructure (V2I) collectively referred to vehicle-to-everything (V2X) communication modes aim to enable data exchanges between vehicles and its surroundings. C-ITS services are one of the promising solutions which main goal is to widely enhance road safety by improving driver awareness about upcoming potential hazards. Through alert notifications, drivers can be assisted to avoid collisions and sudden threats from different actors on the roads.
Since 1999, US Federal Communications Commission (FCC) allocated $75 \mathrm{MHz}$ of spectrum in the $5.9 \mathrm{GHz}$ band to be used by automotive industry for C-ITS. In 2008, ETSI allocated $30 \mathrm{MHz}$ of spectrum in the $5.9 \mathrm{GHz}$ band for ITS. ITS-G5 operates on the frequency band $(5.9 \mathrm{GHz})$ from which its name G5 is derived from.

Currently, many European automotive manufacturers consider ITS-G5/IEEE 802.11p as the most mature short range communication technology for C-ITS. LTE-V2X is another communication technology introduced 3GPP that combines long and short range possibilities. The existence of both technologies has divided the industrial community to two opposing positions, one favouring the existing IEEE 802.11p / ITS-G5 technology, and the other in favour of LTE-V2X as a part of $5 \mathrm{G}$ standardization.

3GPP has already discussed multi-RAT (Radio Access Technology) scenarios in release 16 [1]. In this specification, it is described that LTE-V2X will operate with NR (New Radio)$\mathrm{V} 2 \mathrm{X}$ in order to ensure inter-operability. 3GPP is developing cellular standards for $\mathrm{V} 2 \mathrm{X}$ communications aiming to offer better QoS support, larger coverage, high reliability and low latency. In addition to direct mode communication (V2I, V2V, $\mathrm{I} 2 \mathrm{~V}$ ), this specification proposed a support for wide area communication over a cellular network (V2N) in which different elements that support V2N functionalities communicate with each others using Evolved Packet Switching (EPS).

LTE-V2X is expected to perform the transition path to $5 \mathrm{G}$. But, it still requires further examinations especially that in some vehicular use cases, it is needed to fulfill required latency and reliability in order to guarantee the efficiency of targeted C-ITS services. One of the actual challenges for telecommunication firms is to ensure an adequate choice between both technologies as well as distinguishing scenarios in which a technology can be more suitable compared to another [2].

The main contributions of this paper are divided into two aspects. On one hand, we give a technical insight comparison of both technologies and its associated use cases in ETSI and 3GPP specifications. On the other hand, we aim to identify clear scenarios where the use of one technology yields to better performance results compared to the other technology, and vice versa. Our evaluation is conducted using a realistic simulation platform based on networking and traffic simulators. 
The remainder of this paper is organized as follow. Section II provides an insight about both communication technologies differences. Section III presents a brief summary about the previous work on LTE-V2X and ITS-G5 performance studies. In section [V], we present a performance study for LTE-V2X and ITS-G5 using an urban scenario conditions. Finally, we conclude and we present some perspectives in section $\mathrm{V}$

\section{LTE-V2X AND IEEE 802.11P/ITS-G5 COMMUNICATIONS}

In this section, we will briefly describe the main technical characteristics of both standards ETSI ITS-G5 and 3GPP LTEV2X.

\section{A. ETSI ITS-G5 and C-ITS protocol stack}

ITS-G5 is an access technology conceived by ETSI to support vehicle-to-vehicle, vehicle-to-infrastructure and infrastructure-to-vehicle communications. ITS services messages such as safety or non safety applications are encapsulated into Cooperative Awareness Message (CAM) and Decentralized Environmental Notifications Message (DENM) which are in turn encapsulated into Geo-networking messages and transferred via Basic Transport Protocol (BTP) to the access layer going through the Decentralized Congestion Control (DCC).

The access layer is mainly based on IEEE 802.11 standard and it includes features of IEEE 802.11p. It is composed of the physical layer and the data link layer of the OSI model. The data link layer is divided into two sub-layers: Medium Access Control (MAC) and Logical Link Control (LLC).

The physical layer of ITS-G5 is derived from IEEE 802.11a. In Europe, four specific frequency bands are defined as illustrated in Fig. 1.

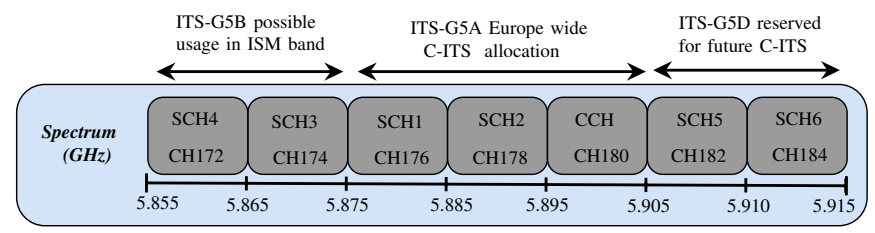

Fig. 1. ITS-G5 spectrum.

For the spectrum bands ITS-G5A and ITS-G5B, the channel spacing is $10 \mathrm{MHz}$. The MAC layer of ITS-G5 have the same scheme as defined in IEEE 802.11-2012. In addition, ITSG5 technology adopts a basic adhoc mode called "Outside the Context of a BSS" (OCB). This mode enables ITSG5 stations to avoid channel scanning, authentication and association operations required for establishing a BSS [3]. ITSG5 station uses a wild-card BSS identifier in order to maintain a direct and immediate message transmission without timeconsuming delay for the exchange of control frames.

\section{B. LTE-V2X}

LTE-V2X is relatively considered as a new technology and it is designed specifically to support vehicular communication scenarios. First version of LTE-V2X was published by 3GPP in 2016 under the umbrella of LTE-release 14 specifications, as an extension of LTE Device-to-Device (D2D) functionality which is standardized in LTE-release 12. LTE-V2X uses sidelink that describes physical channels and which is based itself on LTE uplink waveform. In LTE-V2X, there are two communication radio interfaces: (i) LTE-PC5 also known as LTE side-link (PC5 refers to the radio interface name where the User Equipment (UE) directly communicates with another UE over the direct channel) and (ii) LTE-Uu (UTRAN (Universal Terrestrial Radio Access Network) ue) (The radio interface between the eNodeB and the User Equipment) as shown in Fig. 2 .

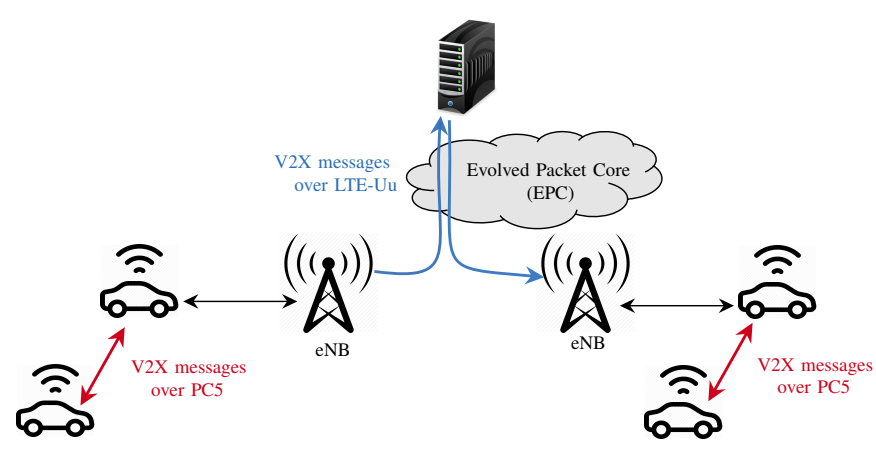

Fig. 2. LTE-V2X architecture.

Direct communication uses the PC5 interface based on Proximity Services Communications (Prose). This interface has enhancements to accommodate high speeds/high Doppler, high vehicle density, improved synchronization and decreased message transfer latency. This mode is suitable for proximate direct communications (hundreds of meters) and for V2V safety applications that require low latency (e.g., ADAS (Advanced Driver Assistance Systems), awareness). For LTEPC5 interface, release 14 3GPP specifications define two communications modes specifically designed for vehicular communications: (i) Mode 3, and (ii) Mode 4.

\section{Mode 3 (In-coverage)}

This mode is also referred to as "base station-scheduled" [4] and "cellular-assisted". In this mode vehicles need to be in the coverage zone of the base station because radio resources of UEs are managed and selected under eNodeB station via control signaling over the $\mathrm{Uu}$ interface. Thus, frequency channel under license by the operator will be used.

Mode 3 resource scheduling algorithms are not detailed in the 3GPP specifications and each operator can develop its own solution. For this mode, according to [5] a possible option is dynamic scheduling in which vehicles request sub-channels to the eNodeB for each packet transmission.

\section{Mode 4 (Out-of-coverage)}

This mode is also known as "pure adhoc V2V" [6] and "autonomously-scheduled" [4]. In this mode vehicles do not need to be in the coverage zone of the base station, because vehicles implement a mechanism for autonomous radio resource 
selection based on sensing before transmission with SemiPersistent Scheduling (SPS). In this mode, license-exempt band will be targeted namely the $5.9 \mathrm{GHz}$ band.

Consequently, LTE-V2X can work both in and out of network coverage. However, network-based communication that uses LTE-Uu interface is supported only when UEs are inside network coverage where UEs are able to receive V2X messages via downlink unicast or uplink broadcast. It also uses the existing LTE Wide Area Network (WAN) and it is suitable for more latency-tolerant use cases (e.g. situational awareness, mobility services).

\section{RELATED WORK}

In this section we will survey some of the existing work on the evaluation of LTE-V2X and ITS-G5.

In paper [7], authors proposed a performance study comparison between IEEE 802.11p and LTE-V2V in both modes 3 and 4. In this paper, authors measured Packet Reception Ratio (PRR) and Update Delay (UD) as performance metrics. Results showed that LTE-V2V (mode 3) can achieve better PRR compared to IEEE 802.11p. In this study, authors evaluated the effect of large packet size on both technologies. They noticed that LTE-V2V (mode 3) outperforms IEEE 802.11p of about $26 \%$ in terms of PRR. They also observed that LTEV2V (mode 4) achieves the worst update delay that reaches values from 1.7 to 3 seconds. Based on their findings, authors concluded that there is no optimal technology that can cover every condition and the choice of a communication technology is closely related to application requirements taking into account communication ranges and packet size.

Paper [8] presented a performance evaluation study of I2V communications using LTE-Advanced (LTE-A) technology. This study adopted multi-lane freeway scenario conditions where the network topology is covered by LTE-A RSU (Road Side Unit) that sends messages to vehicles via downlink mode. Authors focused on studying LTE-A without considering Prose capability (also called (D2D)). In this paper, maximum vehicles number that can be supported by the network was evaluated. As performance metrics, authors chose to analyse Cumulative Distribution Function (CDF) of the SINR. Results showed that around $50 \%$ of vehicles can achieve a SINR of about $15 \mathrm{~dB}$ and $5 \%$ of cell edges vehicles reaches an SINR of $2 \mathrm{~dB}$. They also evaluated network performance in dense scenarios. They used Proportional Fair (PF) scheduler and they observed that cell edge's throughput should be improved since one of the main challenges is to serve the vehicles at the cell edge that require higher number of Physical Resource Blocks (PRB). Based on the obtained results, they concluded about the necessity of developing new resource allocation technique and interference mitigation method in order to achieve the required reliability especially in high network load conditions.

In paper [4], authors focused on investigating the performance of LTE-V2X and IEEE 802.11p in high-density truck-platooning scenario conditions. Authors evaluated CAM message latency and CAM reception rate as performance metrics. For this study, they used Nokia's internal 3GPP system as simulation platform. Furthermore, they compared between IEEE 802.11p, LTE-V2X (mode 4) and LTE-V2X (mode 3). Based on the conducted scenario conditions, they observed that LTE-V2X (mode 3) outperforms IEEE 802.11p in terms of reliability. Results showed that the improvement gain of LTE-V2X (mode 4) over IEEE 802.11p is minor in the case of a short platoon (e.g. up to 5 trucks) and a density of 20 cars $/ \mathrm{km} / \mathrm{lane}$. Long platoons can have more benefits from LTE$\mathrm{V} 2 \mathrm{X}$ due to the better link budget. They concluded that LTE$\mathrm{V} 2 \mathrm{X}$ is more adequate than IEEE $802.11 \mathrm{p}$ for the platooning use case.

Paper [9] proposed a comprehensive overview of LTE-V2X standard. In this paper, authors presented a comparative performance study between IEEE 802.11p and LTE-V2X (mode 4 ) in a fast and slow highway use cases. They observed that LTE-V2X (mode 4) outperforms IEEE 802.11p when the latter is configured with the default data rate of $6 \mathrm{Mb} / \mathrm{s}$. However, IEEE 802.11p improves its performances with a data rate of $18 \mathrm{Mb} / \mathrm{s}$ and even outperforms LTE-V2X (mode 4) under this configuration. Author's conducted study showed that LTEV2X can be an alternative for IEEE 802.11p due to the support of redundant transmission per packet and the infrastructure assistance in (mode 3 ).

In paper [10], authors defined a common scenario to compare IEEE 802.11p and LTE-V2X technologies. Their comparison was conducted using various Modulation Coding Scheme (MCS) possibilities and considering the maximum vehicle density or maximum awareness that can be supported in the same conditions for both technologies. Authors's findings showed that the use of various MCSs enhanced IEEE 802.11p performance especially with longer ranges. For LTE$\mathrm{V} 2 \mathrm{~V}$, authors confirmed the current specification in which the choice of optimal MCS is mainly related to the frame structure and available resources. Authors also showed that $802.11 \mathrm{p}$ standard seems to be efficient in case of short communication range, for instance, they proved that it offers the possibility to support more than one vehicle every 10 meters with an awareness range between 250 and 300 meters. However, in longer ranges, they concluded that communication reliability is highly influenced by collisions which occur due to hidden terminal problem. In the same conditions, they proved that LTE-V2V can also support one vehicle every 10 meters with an awareness range of 500 meters. Consequently, authors confirmed that LTE-V2V is more suitable when larger awareness range is targeted.

In paper [11], authors presented a comparative survey about available communication technologies for Vehicular Ad Hoc Networks (VANETs) and their perspectives towards $5 \mathrm{G}$ path. Firstly, authors gave an insight on available communication standards for Intelligent Transport Systems (ITS) namely IEEE 802.11p, IEEE 802.11bd, LTE-V2X and 5G NR. Authors also highlighted related work dealing with ITS-G5/802.11p and LTE-V2X performance comparison studies. Secondly, they studied performance of both technologies using realistic scenario conditions. Their results allowed them to conclude that LTE-V2X (mode 3) outperforms IEEE 802.11p and LTE-V2X 
(mode 4) since resource allocation is scheduled dynamically via eNodeB and communication range is wider than IEEE 802.11p and LTE-V2X (mode 4).

Different studies concluded that LTE-V2X outperforms IEEE 802.11p without considering the fact that the scheduling mechanism process in LTE V2X (mode 3) can be influenced by other side effects such as usual user services (VoIP, video streaming and so on). Most cited papers did not study the impact of handover procedure of the centralized infrastructure network used by LTE-V2X (mode 3 ). In the next section, we will focus on the impact of concurrent traffic and handover mechanism on the performance of LTE-V2X (mode 3) and compare it to ITS-G5.

\section{Simulation SCENARIOS AND PERFORMANCE EVALUATION OF ITS-G5 AND LTE-V2X (MODE 3)}

We chose to use Artery framework after deeply digging into its features and its implementation that cope with European standardization ITS-G5 for vehicular communications. In fact, Artery offers the possibility to use heterogeneous equipped vehicles with ITS-G5 and LTE protocol stacks [12]. It is also capable to produce a coupled simulation between OMNeT++ and SUMO via Traffic Control Interface (TraCI) as illustrated in Fig. 3 .

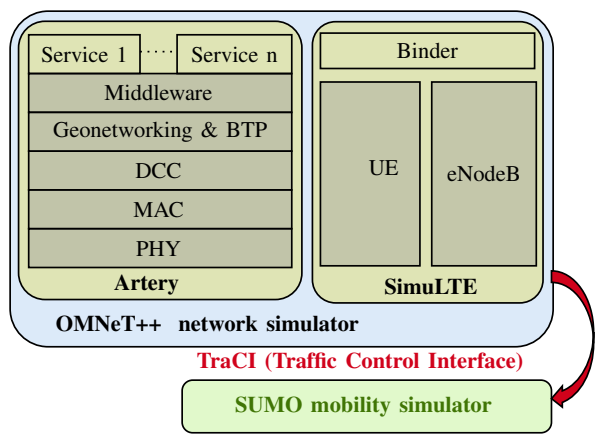

Fig. 3. Simulation architecture

TraCI tool is mainly based on TCP client/server architecture in order to ensure the access to SUMO's simulated objects such as roads and cars. Since 2017, Artery's developers team added LTE support to vehicles [13] through the integration of SimuLTE framework which is a complete representation of an LTE stack within OMNeT++. D2D specific architecture is modeled in SimuLTE. Indeed, MAX-CQI scheduler is adapted for D2D communications. MAX-CQI selects the UEs with the highest CQI to become candidates for scheduling, thereby increasing the overall cell throughput. Currently, D2D capabilities across cell borders "mode 4" are not supported in SimuLTE [14]. As a simulation scenario, we chose to study an alert use case, classified as a cooperative awareness service, that announces an operator vehicle approaching as described in Scoop, Intercor and C-Roads France projects 1 The main idea of this use case is to alert a road user that a

${ }_{1}^{1}$ http://www.scoop.developpement-durable.gouv.fr/en/IMG/pdf/20171013 c-its_french_use_cases_catalog_v4.pdf road operator intervention vehicle is trying to urgently access an area. As a result, alerted road users will be informed in order to facilitate access for the road operator vehicle. Our road topology consists of an urban area where vehicles can be spread in different road sections. A road operator intervention vehicle periodically notifies these vehicles about its crossing using LTE-V2X (mode 3) on Uu interface and ITS-G5 via $\mathrm{V} 2 \mathrm{~V}$ communication mode. As performance metrics, we chose to measure End-to-End (E2E) delay which is defined as the needed time for the destination service to get the packet generated by the source service, Channel Busy Ratio (CBR) and percentage of Cell Block Utilization (CBU). Note that in our evaluation study, we are only interested in the wireless part of both technologies. Thus, our evaluation does not take into account the impact of the wired infrastructure of neither LTE nor ITS-G5. Table I illustrates the simulation parameters.

TABLE I

SIMULATION PARAMETERS

\begin{tabular}{|c|c|}
\hline \multicolumn{2}{|c|}{ Traffic mobility parameters } \\
\hline Vehicles speed $[\mathrm{km} / \mathrm{h}]$ & 30 \\
\hline Number of vehicles & [30..90] \\
\hline Car-following model & Krauss \\
\hline \multicolumn{2}{|c|}{ Alert service configuration } \\
\hline Message size [bytes] & 800 \\
\hline Sending frequency $[\mathrm{Hz}]$ & 10 \\
\hline \multicolumn{2}{|c|}{ ITS-G5/802.11p related parameters } \\
\hline Bit rate $[\mathrm{Mbps}]$ & 6 \\
\hline EDCA Access Category (AC) & $A C_{V O}$ \\
\hline Transmission power [mw] & 12 \\
\hline Sensitivity $[\mathrm{dBm}]$ & -85 \\
\hline Propagation model & Two rays interference \\
\hline \multicolumn{2}{|c|}{ LTE-Uu related parameters } \\
\hline Cell type & Urban macro cell \\
\hline Scheduling mechanism & MAX-CQI \\
\hline D2D Channel Quality Indicator (CQI) & 7 \\
\hline Carrier frequency $(\mathrm{GHz})$ & 2.1 \\
\hline Antenna Gain $[\mathrm{dB}]$ & $\mathrm{UE}(0)-\mathrm{eNB}(18)$ \\
\hline TX power $[\mathrm{dBm}]$ & UE (26) - eNB (46) \\
\hline Noise figure & $\mathrm{UE}(7)-\mathrm{eNB}(5)$ \\
\hline Channel bandwidth [Hz] & 10 \\
\hline
\end{tabular}

Table $\mathrm{II}$ gives the performance requirements of $\mathrm{V} 2 \mathrm{X}$ use cases as indicated in 3GPP TR 22.186 V15.0.0 reference document.

TABLE II

PERFORMANCE REQUIREMENTS OF SOME V2X USE CASES FROM 15

\begin{tabular}{c||c||c}
\hline \hline Use Case type & E2E latency & Reliability (\%) \\
\hline Cooperative awareness & $100 \mathrm{~ms}$ & $90-95$ \\
\hline Traffic efficiency & $>1 \mathrm{sec}$ & $<90$ \\
\hline Extended sensors & $3-100 \mathrm{~ms}$ & $90-99.99$ \\
\hline Advanced driving & $3-100 \mathrm{~ms}$ & 99.99 \\
\hline Platooning & $10-25 \mathrm{~ms}$ & $90-99.99$ \\
\hline \hline
\end{tabular}

\section{A. Scenario 1: realistic data traffic effects on ITS alert service}

In this first scenario, we are interested in evaluating the impact of additional traffic on the performance of LTE-V2X. The LTE network can be used for transmitting various applications traffic not related to C-ITS applications. This traffic, 
mostly generated by mobile devices of drivers or other users in the area, will share the network resources with C-ITS traffic. In our scenario, we only considered one mobile device per vehicle. All users are downloading video streaming data during simulation, and send alert notifications periodically. Table III summarizes the video streaming application parameters. These parameters are based on collected data of an average typical data traffic usage per day and it represent a realistic network traffic load [16].

TABLE III

PARAMETERS OF VIDEO STREAMING DATA

\begin{tabular}{c|c}
\hline \hline Video size & $30 \mathrm{MiB}$ \\
\hline \hline Packet length & $1000 \mathrm{~B}$ \\
\hline \hline Sending interval & $20 \mathrm{~ms}$ \\
\hline \hline Simulation time & $110 \mathrm{~s}$ \\
\hline \hline
\end{tabular}

\section{A.1 Ideal LTE RF conditions scenario:}

For this scenario, excellent RF conditions are ensured during the entire simulation. We set the number of vehicles to 20 and we kept one operator vehicle at the end of the flow of vehicles (number 19) that sends alert notifications to the rest of the vehicles in order to facilitate its way as shown in Fig. 4

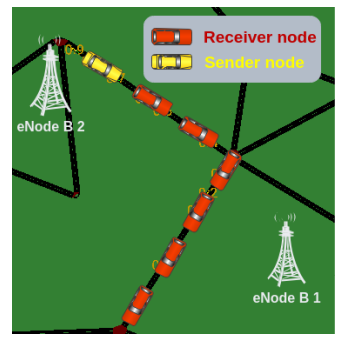

Fig. 4. Ideal RF conditions

Fig. 5 illustrates E2E delay per receiving node. We observe that ITS-G5 achieves a delay approximately ten times lower than LTE-V2X. Using ITS-G5 nodes (0, 1 and 2) which illustrate vehicles on the top do not receive the alert message due to coverage limitation.

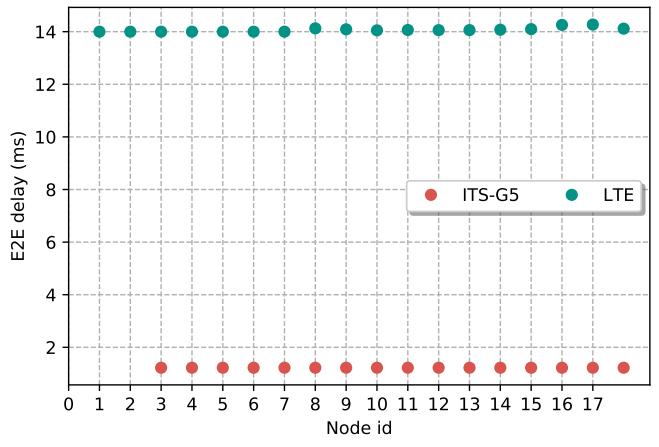

Fig. 5. E2E delay per receiving node
Using LTE-V2X all nodes receive the alert message with an E2E delay of $14 \mathrm{~ms}$ which still meets latency requirements as shown in table II Indeed, generating simultaneous video traffic have a direct impact of the E2E delay of LTE-V2X.

\section{A.2 Heterogeneous LTE RF conditions scenario:}

In this scenario, we set five operator vehicles in different areas that periodically send alert notifications to all users in their proximity in order to facilitate their passage as shown in Fig. 6.

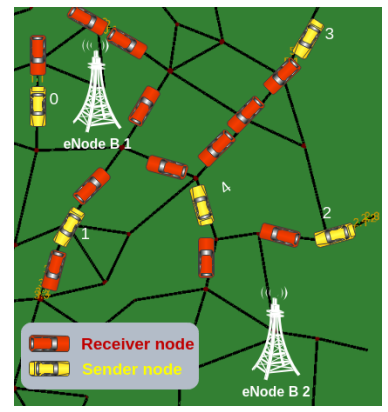

Fig. 6. Heterogeneous RF conditions scenario

Fig. 7 shows average time ratio of RF conditions for nodes during simulation. These percentages are calculated based on the SINR values of nodes that allows us to classify RF conditions based on table IV

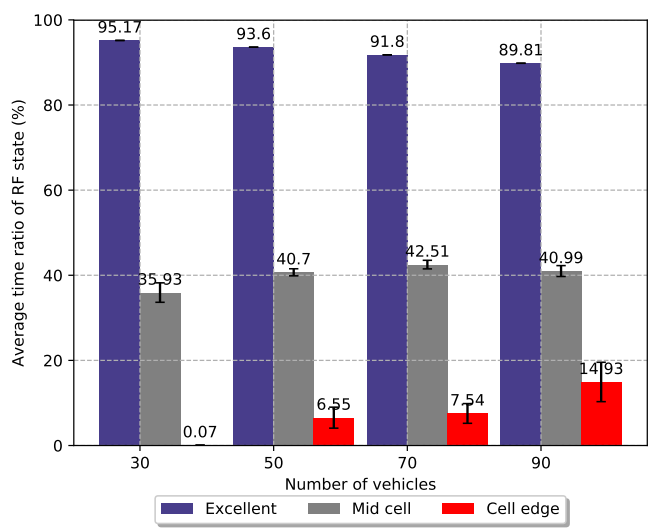

Fig. 7. Time ratio indicating LTE RF conditions

As shown in Fig. 7, nodes reach cells edges for a number of vehicles between 50 and 90. This is the main reason that impact the increase of E2E delay and explains the great variations for 70 And 90 vehicles scenarios.

TABLE IV

RF CONDITIONS VS LTE KPIS FROM [17]

\begin{tabular}{c||c||c}
\hline \hline RF conditions & SINR $(\mathrm{dB})$ & RSRP $(\mathrm{dBm})$ \\
\hline Excellent & $\geq 20$ & $\geq-80$ \\
\hline Good & 13 to 20 & -80 to -90 \\
\hline Mid cell & 0 to 13 & -90 to -100 \\
\hline Cell edge & $\leq 0$ & $\leq-100$ \\
\hline \hline
\end{tabular}


Fig. 8 shows E2E delay evolution for different number of vehicles. We observe that delay increases to reach almost 240 ms using LTE-V2X (mode 3) in the case of a dense scenario of 90 vehicles. With ITS-G5, we obtain a lower delay than that of LTE-V2X (mode 3), since it is dedicated to C-ITS services.

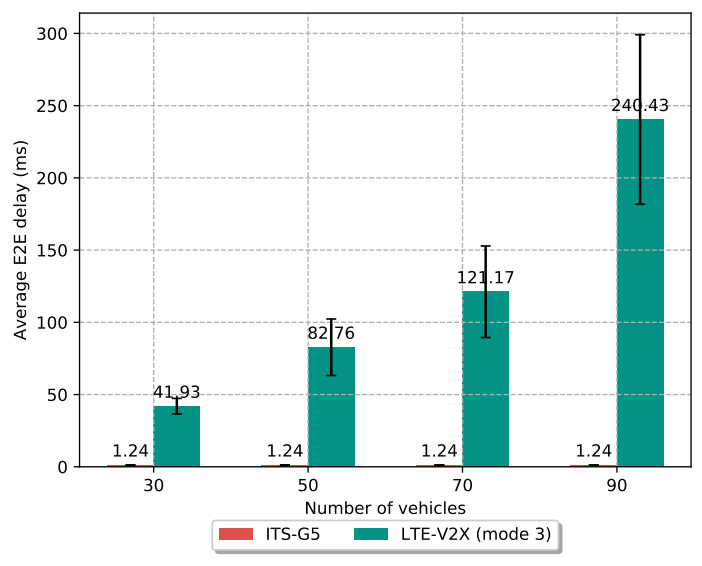

Fig. 8. Average E2E delay

In Fig. 9, we show alert reception ratio. This metric is calculated as a ratio between the number of nodes from which a node receives the message and the number of sender nodes. For instance, in case of scenario of 30 vehicles, using ITSG5, a node receives messages from an average of almost $62 \%$ of nodes in its proximity. However, using LTE-V2X (mode 3), a node receives notifications from almost $91 \%$ of sender nodes. LTE-V2X (mode 3) outperforms ITS-G5 due to its wider coverage. Indeed, vehicles receive more alerts using LTE-V2X compared to ITS-G5.

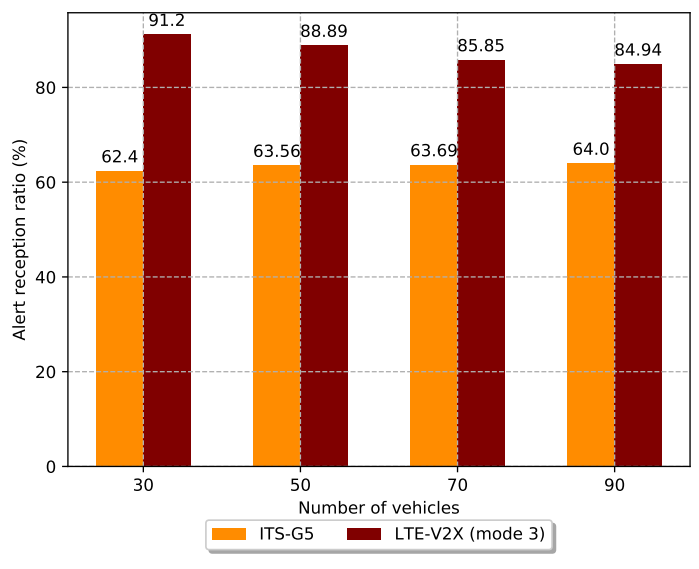

Fig. 9. Alert reception ratio

Fig. 10 shows respectively the channel busy ratio of ITS-G5 and cell block utilization of eNB1 and eNB2 in downlink (DL) and uplink (UL). We observe that these two metrics increase with the increase of number of vehicles which is an expected result that explains the increase of E2E delay in Fig. 8
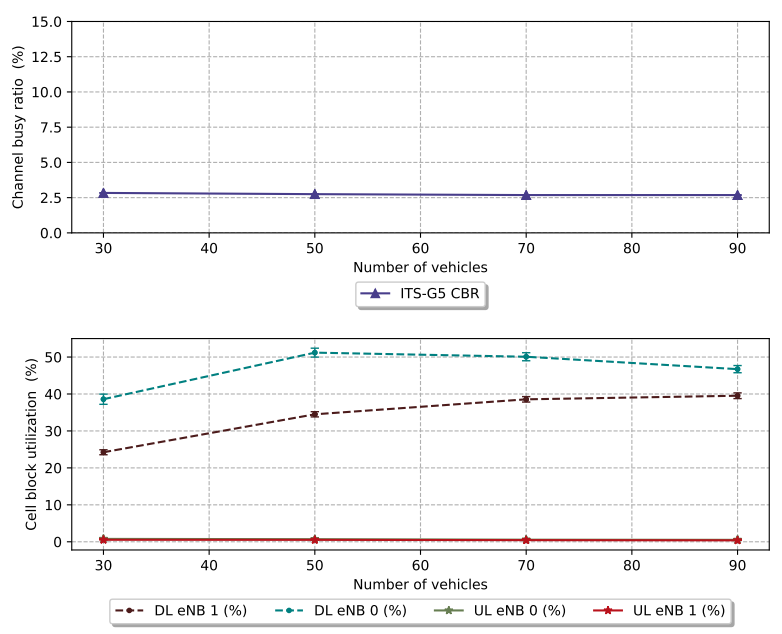

Fig. 10. Channel busy ratio and cell block utilization per number of vehicles

\section{B. Scenario 2: handover impact on ITS safety service}

In this scenario, we investigate handover effect on ITS alert service. We set the number of vehicles to 10 in which vehicle number 4 sends messages to vehicles to alert them for facilitating its way. These vehicles cross both cells areas. Sender vehicle "node 4" is inserted at time at instant $11 \mathrm{~s}$, this is why data is recorded starting at second 11 .
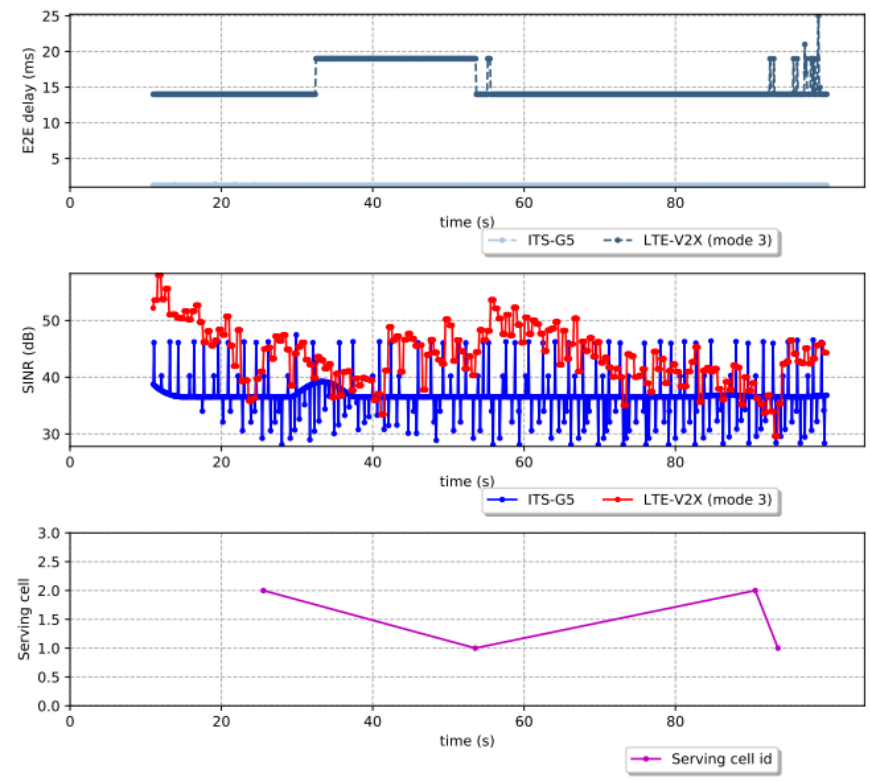

Fig. 11. SINR measurements, E2E delay and serving cell for CQI=3

In this case, handover latency is set to a mean of a random variable of $50 \mathrm{~ms}$. In 3GPP technical report (release 14) [18], minimum latency is calculated during handover and it is around values that scale between $45.5 \mathrm{~ms}$ and $49.5 \mathrm{~ms}$. Fig. 11 shows the SINR of node "1" which is a receiver node. For ITS-G5, SINR scales between $28 \mathrm{~dB}$ and $47.4 \mathrm{~dB}$. Using 
LTE-V2X, it varies between $29.5 \mathrm{~dB}$ and $57.9 \mathrm{~dB}$. Fig. 11 also illustrates E2E delay and serving id evolution during the simulation scenario using a CQI equal to 3 . We observe that E2E delay increases after switching to eNB2 to reach about 20 ms. Fig. 12 shows E2E delay of node "1" using a CQI equal to 15 . In this case, we observe that SINR scales between 30.4 $\mathrm{dB}$ and $63.4 \mathrm{~dB}$. The change of CQI directly influences SINR since this latter is a direct indicator of bit rate. For $\mathrm{CQI}=15$, we observe that E2E delay is less influenced by handover process.
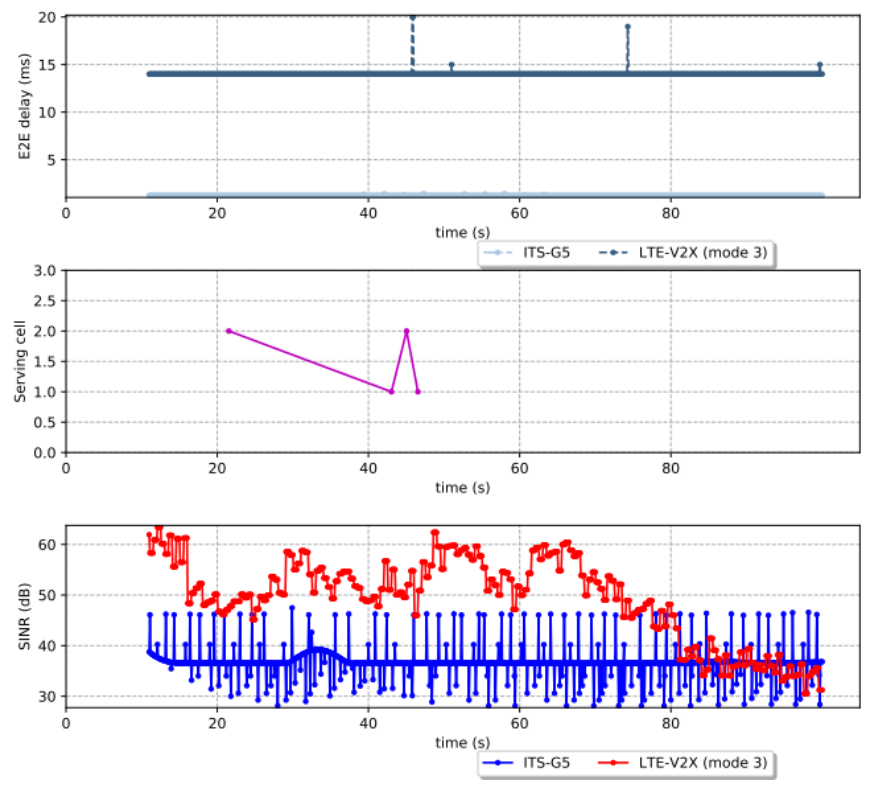

Fig. 12. SINR measurements, E2E delay and serving cell for $\mathrm{CQI}=15$

The effect of handover process on E2E delay mainly depends on the reception of control messages by a UE from eNB. Indeed, the UE periodically receives this control message that contains information about signal strength of target cell. Thus, the UE checks if it can attach to a better eNB considering both SINR and hysteresis. For both cases, the obtained E2E delay meets the delay requirement of an ITS safety service since it is blow $100 \mathrm{~ms}$.

\section{CONCLUSION AND FUTURE WORK}

In this paper, ITS-G5 and LTE-V2X (mode 3) technologies are studied. We defined scenarios where we compare both technologies performances in terms of E2E delay and RF conditions through measuring SINR and RSRP. ITS-G5 is dedicated to vehicular communications whereas LTE-V2X (mode 3) is expected to operate in the frequency band of mobile operators where other applications (such as video streaming and VoIP etc.) can be scheduled. This aspect can impact the performance of ITS services. Moreover, handover procedure can negatively influences ITS services performance in terms of E2E delay. In ITS-G5 standard, association and authentication mechanisms are disabled with OCB mode, this is considered as one of the advantages of ITS-G5 over LTE-V2X (mode 3). The choice of a technology instead to another is closely related to the use case conditions and ITS service quality of service requirements. We used a network simulation platform that emulates communication protocols of both technologies including the wireless medium and a traffic simulator. The conducted scenarios of our study indicate the need of developing a decision technique that switches between both technologies depending on the state of their network. This idea will be tackled in our future work. Our main goal will be to ensure the selection of an adequate technology, according to a set of well-defined criteria considering C-ITS service performance requirements in terms of latency and reliability.

\section{REFERENCES}

[1] "3GPP TR 22.886 V16.1.1; technical specification group services and system aspects; study on enhancement of 3GPP support for 5G V2X services (release 16)," Septembre 2018.

[2] A. Filippi, K. Moerman, G. Daalderop, P. D. Alexander, F. Schober, and W. Pfliegl, "Ready to roll: Why 802.11 p beats LTE and 5G for V2X," NXP Semiconductors, Cohda Wireless and Siemens White Paper, 2016.

[3] A. Festag, "Cooperative intelligent transport systems standards in Europe," IEEE Communications Magazine, 2014.

[4] V. Vukadinovic, K. Bakowski, P. Marsch, I. D. Garcia, H. Xu, M. Sybis, P. Sroka, K. Wesolowski, D. Lister, and I. Thibault, "3GPP C-V2X and IEEE $802.11 \mathrm{p}$ for Vehicle-to-Vehicle communications in highway platooning scenarios," Ad Hoc Networks, vol. 74, pp. 17-29, 2018.

[5] G. Nardini, A. Virdis, C. Campolo, A. Molinaro, and G. Stea, "Cellularv2x communications for platooning: Design and evaluation," Sensors, vol. 18 , no. 5 , p. $1527,2018$.

[6] E. A. M. A. (ACEA), "Frequency bands for V2X," vol. 7, 2018.

[7] G. Cecchini, A. Bazzi, B. M. Masini, and A. Zanella, "Performance comparison between ieee $802.11 \mathrm{p}$ and LTE-V2V in-coverage and out-of-coverage for cooperative awareness," in 2017 IEEE Vehicular Networking Conference (VNC). IEEE, 2017, pp. 109-114.

[8] P. Luoto, M. Bennis, P. Pirinen, S. Samarakoon, K. Horneman, and M. Latva-aho, "System level performance evaluation of LTE-V2X network," in European Wireless 2016; 22th European Wireless Conference; Proceedings of. VDE, 2016, pp. 1-5.

[9] R. Molina-Masegosa and J. Gozalvez, "LTE-V for sidelink 5G V2X vehicular communications: A new 5G technology for short-range vehicleto-everything communications," IEEE Vehicular Technology Magazine, vol. 12, no. 4, pp. 30-39, 2017.

[10] A. Bazzi, B. M. Masini, A. Zanella, and I. Thibault, "On the performance of IEEE $802.11 \mathrm{p}$ and LTE-V2V for the cooperative awareness of connected vehicles," IEEE Transactions on Vehicular Technology, vol. 66, no. 11 , pp. 10419-10 432, 2017.

[11] A. Bazzi, G. Cecchini, M. Menarini, B. M. Masini, and A. Zanella, "Survey and perspectives of vehicular Wi-Fi versus sidelink cellularV2X in the 5G Era," Future Internet, vol. 11, no. 6, p. 122, 2019.

[12] R. Riebl, G. Nardini, and A. Virdis, "Simulating LTE-Enabled vehicular communications," in Recent Advances in Network Simulation. Springer, 2019, pp. 407-423.

[13] H.-J. Günther, J. Timpner, M. Wegner, R. Riebl, and L. Wolf, "Extending a holistic microscopic ivc simulation environment with local perception sensors and LTE capabilities," Vehicular Communications, vol. 9, pp. 211-221, 2017.

[14] G. Nardini, A. Virdis, and G. Stea, "Modeling network-controlled Device-to-Device communications in simuLTE," Sensors, vol. 18, no. 10, p. 3551, 2018.

[15] "3GPP TR 22.186 V15.0.0: Service requirements for enhanced V2X scenarios (release 15), 3GPP Std.” March 2017.

[16] L. korowajczuk, "How to dimension user traffic 4G networks," CelPlan international, inc, 2014.

[17] S. A. Khan, M. Asshad, A. Kavak et al., "A Power Control Algorithm (PCA) and software tool for femtocells in LTE-A networks," Sakarya University Journal of Science, vol. 22, no. 4, pp. 1124-1129, 2018.

[18] "3GPP Technical Report 36.881 V1.0.0 3rd generation partnership project; technical specification group radio access network; evolved universal terrestrial radio access (e-utra); study on latency reduction techniques for LTE (release 14)," Mai 2016. 